\title{
DNA methylation signatures associated with prognosis of gastric cancer
}

Jin Dai ${ }^{1,2}$, Akihiro Nishi ${ }^{2}$, Zhe-Xuan $\mathrm{Li}^{1}$, Yang Zhang ${ }^{1}$, Tong Zhou', Wei-Cheng You' ${ }^{1}$, Wen-Qing $\mathrm{Li}^{1,3^{*}+}$ and Kai-Feng Pan $^{1 *+}$

\begin{abstract}
Background: Few studies have examined prognostic outcomes-associated molecular signatures other than overall survival (OS) for gastric cancer (GC). We aimed to identify DNA methylation biomarkers associated with multiple prognostic outcomes of GC in an epigenome-wide association study.

Methods: Based on the Cancer Genome Atlas (TCGA), DNA methylation loci associated with OS ( $n=381)$, diseasespecific survival (DSS, $n=372$ ), and progression-free interval ( $\mathrm{PFI}, n=383$ ) were discovered in training set subjects (false discovery rates $<0.05$ ) randomly selected for each prognostic outcome and were then validated in remaining subjects ( $P$-values $<0.05$ ). Key CpGs simultaneously validated for OS, DSS, and PFI were further assessed for diseasefree interval (DFI, $n=247)$. Gene set enrichment analyses were conducted to explore the Gene Ontology and Kyoto Encyclopedia of Genes and Genomes pathways simultaneously enriched for multiple GC prognostic outcomes. Methylation correlated blocks (MCBs) were identified for co-methylation patterns associated with GC prognosis. Based on key CpGs, risk score models were established to predict four prognostic outcomes. Spearman correlation analyses were performed between key CpG sites and their host gene mRNA expression.
\end{abstract}

Results: We newly identified DNA methylation of seven CpGs significantly associated with OS, DSS, and PFI of GC, including cg10399824 (GRK5), cg05275153 (RGS12), cg24406668 (MMP9), cg14719951(DSC3), and cg25117092 (MED12L), and two in intergenic regions (cg11348188 and cg11671115). Except cg10399824 and cg24406668, five of them were also significantly associated with DFI of GC. Neuroactive ligand-receptor interaction pathway was suggested to play a key role in the effect of DNA methylation on GC prognosis. Consistent with individual CpGlevel association, three MCBs involving cg11671115, cg14719951, and cg24406668 were significantly associated with multiple prognostic outcomes of GC. Integrating key CpG loci, two risk score models performed well in predicting GC prognosis. Gene body DNA methylation of cg14719951, cg10399824, and cg25117092 was associated with their host gene expression, whereas no significant associations between their host gene expression and four clinical prognostic outcomes of GC were observed.

Conclusions: We newly identified seven CpGs associated with OS, DSS, and PFI of GC, with five of them also associated with DFI, which might inform patient stratification in clinical practices.

\footnotetext{
* Correspondence: wenqing_li@bjmu.edu.cn; pankaifeng2002@yahoo.com

${ }^{+}$Wen-Qing Li and Kai-Feng Pan contributed equally to this work.

${ }^{1}$ Key Laboratory of Carcinogenesis and Translational Research (Ministry of Education/Beijing), Department of Cancer Epidemiology, Peking University Cancer Hospital \& Institute, 52 Fucheng Rd, Haidian District, Beijing 100142, People's Republic of China

Full list of author information is available at the end of the article
}

(c) The Author(s). 2021 Open Access This article is licensed under a Creative Commons Attribution 4.0 International License, which permits use, sharing, adaptation, distribution and reproduction in any medium or format, as long as you give appropriate credit to the original author(s) and the source, provide a link to the Creative Commons licence, and indicate if changes were made. The images or other third party material in this article are included in the article's Creative Commons licence, unless indicated otherwise in a credit line to the material. If material is not included in the article's Creative Commons licence and your intended use is not permitted by statutory regulation or exceeds the permitted use, you will need to obtain permission directly from the copyright holder. To view a copy of this licence, visit http://creativecommons.org/licenses/by/4.0/. The Creative Commons Public Domain Dedication waiver (http://creativecommons.org/publicdomain/zero/1.0/) applies to the data made available in this article, unless otherwise stated in a credit line to the data. 
Keywords: Bioinformatics, Biomarkers, Epigenetics, Gastric cancer, Heterogeneity, Methylation, Precision medicine, Prognosis, Survival, The Cancer Genome Atlas

\section{Introduction}

Gastric cancer (GC) is the fifth most common cancer and third leading cause of cancer deaths worldwide [1]. A considerable proportion of GC patients are diagnosed at advanced stages and their prognosis remains poor [2]. Moreover, GC patients with same clinical stage may develop diverse prognostic outcomes due to the epigenetic or genetic host heterogeneities. Hence, identifying molecular signatures to predict GC prognosis would be warranted for tailored clinical procedures. However, established molecular biomarkers which could predict the prognosis of GC are still very limited.

DNA methylation is a covalent chemical modification, which results in the addition of a methyl $\left(\mathrm{CH}_{3}\right)$ group at the carbon 5 position of the cytosine ring [3]. Alterations in DNA methylation, possessing several merits, such as high frequency in tumor, easy detection, and stability in fixed samples over time, have been considered as promising targets for developing prognostic biomarkers [4]. Based on the Cancer Genome Atlas (TCGA), several studies have identified DNA methylation-based biomarkers associated with overall survival (OS) of GC patients, with three of them based on differentially methylated genes between GC tumor and normal tissue [5-7] and the other one not appropriately account for multiple hypothesis testing [8]. In addition, although OS is the most clinically relevant endpoint with the least equivocal definition, solely using OS as the prognostic endpoint may not be sufficient to mirror all perspectives of tumor prognosis, as patient deaths may be due to GC or other causes [9]. Disease-specific survival (DSS) therefore would possess greater relevance to GC-specific biology and therapeutic impact. In addition, given the relatively short follow-up period of GC (median follow-up time $=14$ months) in TCGA, the number of deaths at the end of follow-up may be insufficient to reveal a difference in deaths among comparison groups $[10,11]$. Progression-free interval (PFI) and disease-free interval (DFI) have therefore been considered as better choices of surrogate clinical endpoints. To our best knowledge, few studies have identified DNA methylation-based biomarkers associated with prognostic outcomes other than OS of GC patients.

In the present epigenome-wide association study, we comprehensively examined the association between DNA methylation and four prognostic outcomes of GC, including OS, DSS, PFI, and DFI of GC patients in TCGA.

\section{Materials and methods}

\section{Data sources and preprocessing}

DNA methylation profile (IDAT files) from 395 GC patients were obtained using $\mathrm{R} /$ Bioconductor package
TCGAbiolinks [12]. In TCGA, DNA methylation of cancer tissue from all patients were assayed using the Infinium HumanMethylation450 BeadChip arrays. A TCGA online tool was used to estimate batch effect (https:// bioinformatics.mdanderson.org/BatchEffectsViewer/), which would be presented if the dispersion separability criterion (DSC) value was greater than 0.5 and the DSC $P$-value was less than 0.05 . Data importation, annotation, and quality control were conducted in the R/Bioconductor package minfi [13]. We used R/Bioconductor package SeSAMe to perform background correction and dyebias correction [14, 15]. In brief, normal-exponential using out-of-band probes (NOOB) background correction was utilized to account for additive error to the measure of signal intensities due to background fluorescence. Non-linear dye-bias correction was performed to control for the different average intensities in the red and green channels of Type II probes. In addition, Pvalue with out-of-band (OOB) array hybridization (POOBAH) method was applied to address artifacts which reflected failed hybridization to target DNA respectively. For probe filtering, we excluded probes for which the detection $P$-value was bigger than 0.05 for more than $10 \%$ of the remaining samples, those on the sex chromosomes, those mapped to multiple regions in the genome, and those harboring known singlenucleotide polymorphisms with a minor allele frequency greater than 5\% [16]. After data preprocessing, a total of 411,408 CpG sites were kept in our analysis, including 163,453 in promoter, 139,213 in gene body, 14,896 in 3'UTR, and 93,846 in intergenic regions.

Demographical and clinical characteristics of GC patients, including age at initial pathologic diagnosis, gender, ethnicity, histological grade, anatomic region, family history of GC, Helicobacter pylori infection, radiation therapy, and the tumor, lymph nodes, and metastasis (TNM) stage were obtained using R/Bioconductor package TCGAbiolinks [12]. We further imputed missing ethnicities using R/Bioconductor package SeSAMe [15]. Four prognostic outcomes, including OS, DSS, PFI, and DFI of GC, were derived from those defined in a previous study [11]. In short, using the date of diagnosis of primary GC as the beginning date, OS was defined as the period until the date of death from any cause; DSS was defined as the period until the date of GC-specific death; PFI was defined as the period until the date of the first occurrence of a new GC; DFI was defined as the period until the date of the first new GC progression event subsequent to the ascertainment of a patient's 
disease-free status after their initial GC diagnosis and treatment. The GC-free status of patients must be confirmed in order for them to be qualified for calculating DFI.

\section{Identification of key CpG sites associated with four clinical endpoints}

For all 411,408 CpG sites, we first examined the association between DNA methylation of each individual CpG site and OS of GC. GC patients with information on OS were randomly divided into training and validation sets (50:50). For CpG sites significantly associated with OS in the training set (false discovery rate $(F D R)<0.05$ considering multiple comparisons) [17], we sought to replicate the associations in the validation sets $(P<0.05)$. For those replicated CpG loci, associations with DSS and PFI of GC were then examined. GC patients with information on DSS or PFI were randomly divided into training and validation sets (50:50) for the assessment of each specific outcome. We then selected key CpG sites significantly associated with OS, DSS, and PFI in the respective training and validation sets, which were further assessed for the association with DFI. Due to the difficulty of ascertaining GC-free status of patients after their initial diagnosis and treatment, a large body of patients, especially for those in stage IV, were excluded in DFI analysis. We therefore did not seek to divide it into training and validation sets for statistical power consideration. Cox proportional hazard analyses were performed to calculate hazard ratios (HRs) and 95\% confidence intervals (CIs) for the association between each individual CpG site and clinical endpoint, adjusting for age at initial pathologic diagnosis (continuous) and gender. For those CpG sites significantly associated with clinical endpoints, sensitivity analyses were conducted to test the robustness of the associations by additionally adjusting for other potential confounders, including ethnicity (Asian, Black or African American, or White), TNM stage (T1-2, T3-4, or unknown; N0-1, N2-3, or unknown; M0, M1, or unknown), histological grade (G1-2, G3, or unknown), anatomic neoplasm region (antrum/distal, cardia/proximal, fundus/body, gastroesophageal junction, or unknown), radiation therapy (yes, no, or unknown), H. pylori infection (yes, no, or unknown), and family history of GC (yes, no, or unknown). We did not find violations of proportional hazard assumptions for all Cox proportional hazard models.

\section{Gene set enrichment analysis}

Gene set enrichment analyses (GSEA) were conducted to explore the Gene Ontology (GO) and Kyoto Encyclopedia of Genes and Genomes (KEGG) pathways which simultaneously enriched for multiple GC prognostic outcomes using DNA methylation data $[18,19]$.
The analyses were conducted utilizing functional class scoring approach, which overcomes the drawbacks of over-representation analysis, another commonly used method for GSEA [20]. As most of the CpG sites significantly associated with GC prognosis in our study were in gene body, we focused on CpG sites located in gene body region only for GSEA. All preprocessed CpG sites in gene body region were ranked based on their Cox regression $P$-values with $\mathrm{GC}$ prognosis. Gene set level $P$ values were computed through permutation using $R /$ Bioconductor package methylGSA [21]. The number of CpG sites located in one gene was adjusted in the logistic model to avoid length bias. Gene sets were defined as those containing at least 10 genes and at most 500 genes. FDR less than 0.05 was set as the criteria defining significantly enriched GO or KEGG terms.

\section{Identification of methylation correlated blocks (MCBs) based on key CpGs}

In addition to individual CpG loci-level analyses, we also examined the association for a cluster of $\mathrm{CpG}$ sites located in the same genomic region based on the concept of genetic linkage disequilibrium. To investigate whether key CpG sites had co-methylation patterns with their adjacent CpG sites and whether these patterns were associated with GC prognosis, Pearson correlation analyses were conducted between DNA methylation level of key CpG sites and their adjacent loci positioned within their flanking areas of one kilobase up- or downstream. Pearson correlation coefficients less than 0.5 indicated boundaries of uncorrelated methylation [22]. For adjacent loci that were not separated by boundaries, we continued examining the correlation between DNA methylation level of these loci and their neighboring loci located within their flanking areas. All CpG sites not separated by the boundary were combined into methylation correlated blocks (MCBs) [22]. The mean $\beta$ values of all CpG sites within one MCB was defined as the methylation value of that MCB. The R/Bioconductor co-MET package was utilized to visualize the co-methylation patterns of identified MCBs [23]. We then examined the association between those identified MCBs and four clinical endpoints using Cox regression analyses adjusting for age at initial pathologic diagnosis (continuous) and gender. For significant MCBs, sensitivity analyses were also performed, similar to those conducted for individual $\mathrm{CpG}$ sites.

\section{Establishment of two risk score models}

Incorporating key CpG sites associated with GC prognosis, risk score models to predict the risk of specific prognostic outcomes were constructed, which were defined as the sum of auto-scaled DNA methylation level of each CpG weighted by the regression coefficients obtained from the Cox regression model in the whole dataset: 
Risk score $=\beta_{1} X_{1}+\beta_{2} X_{2}+\beta_{3} X_{3}+\ldots \ldots+\beta_{n} X_{n}$,

GC patients were categorized into low-, median-, and high- risk score groups based on the tertile of their risk scores. Kaplan-Meier survival curves were drawn and log-rank tests were performed to compare the survival probabilities among different risk score groups.

\section{Correlation between DNA methylation and mRNA expression}

To explore the potential regulating effect of DNA methylation on host gene expression, Spearman correlation analyses were performed between DNA methylation of those key CpG sites and their host gene mRNA expression. Normalized encoding genes' mRNA expression data (legacy data) based on RNA-Seq (Illumina RNA-Seq HiSeq platform) was downloaded and preprocessed using R/Bioconductor package TCGAbiolinks [12]. Both mRNA expression level and DNA methylation level ( $\beta$ value) were auto-scaled before down-stream analysis. $P$-value $<0.05$ was set as the criteria for the significant correlation. For those genes whose DNA methylation was correlated with their mRNA expression, the association of their mRNA expression with four clinical endpoints of $\mathrm{GC}$ were then examined using Cox regression analysis.

All analyses were performed and visualized using $R$ version 4.0.2.

\section{Results}

\section{Major characteristics of GC patients in TCGA}

In the present study, we included GC patients having both DNA methylation profiles and intact follow-up information of OS $(n=381)$, DSS $(n=372)$, PFI $(n=383)$, and DFI $(n=247)$. During the follow-up, 149, 99, 137, and $46 \mathrm{GC}$ patients developed corresponding clinical endpoints of OS (death from any cause), DSS (GC-specific death), PFI (the first occurrence of a new GC event), and DFI (the first new GC progression event), respectively. Major characteristics of included GC patients were shown in Table 1.

\section{Identification of key $\mathrm{CpG}$ sites associated with four clinical endpoints}

According to TCGA Batch Effect Viewer, no significant batch effect was observed (DSC $=0.307$, DSC $P$-value $=5$ $\times 10^{-4}$ ). After data preprocessing, 411,408 CpG sites were kept in our analysis. By performing the Cox regression analyses for all preprocessed CpGs, we identified a total of 488 CpG sites significantly associated with OS (FDR $<0.05$ in the training set and $P$-value $<0.05$ in the validation set). Among them, seven key CpG sites were simultaneously associated with DSS and PFI in both training (FDR $<0.05)$ and validation sets $(P$-value $<0.05)$, including cg10399824 (G protein-coupled receptor kinase 5, GRK5), cg05275153 (regulator of G protein signaling 12, RGS12), cg24406668 (matrix metallopeptidase 9, MMP9), cg14719951 (desmocollin 3, DSC3), and cg25117092 (mediator complex subunit $12 \mathrm{~L}, M E D 12 L / M E D 12)$ and two CpG loci (cg11348188 and cg11671115) located within intergenic regions. Of them, DNA hypermethylation of three $\mathrm{CpG}$ sites, including cg11348188 and cg11671115 in intergenic regions, and cg25117092 in gene body was significantly associated with unfavorable OS, DSS, and PFI of GC, whereas DNA hypermethylation of cg05275153, cg14719951, cg10399824, and cg24406668 in gene body was significantly associated with favorable OS, DSS, and PFI of GC. These CpG loci were further assessed for DFI of GC. Except for cg10399824 and cg24406668, DNA methylation level of the other five CpG sites was also significantly associated with DFI. The directions of associations between seven key CpG sites and all four clinical endpoints remained consistent (Table 2).

\section{GSEA based on CpGs within gene body}

Since five key CpG sites that had their host gene information were all located in gene body, GSEA was conducted exclusively based on all CpG sites located in gene body to elucidate the biological mechanisms of gene body DNA methylation on GC prognosis. A total of five GO terms were enriched in all four clinical endpoints according to their original $P$-values, but none remained simultaneously significant for all four endpoints after multiple comparison adjustment. Four KEGG pathways were simultaneously enriched in OS, DSS, and PFI, with one pathway involving neuroactive ligand-receptor interaction remaining consistently significant even after multiple comparison adjustment (Table 3, Supplementary Table S1).

\section{Identification of MCBs associated with GC prognosis}

Investigating the potential co-methylation patterns focusing on seven key CpG sites, we identified three MCBs, including one consisting of cg11671115 and cg19989498 in the intergenic region, another containing cg14719951 and 12 adjacent CpG sites in DSC3 gene body, and the third one including cg24406668 and seven adjacent $\mathrm{CpG}$ sites in MMP9 gene body. Consistent with individual CpG-level association, DNA methylation of these three MCBs was significantly associated with multiple prognostic outcomes of GC (Fig. 1).

\section{Establishment of two risk score models}

Based on DNA methylation of the seven key CpG sites associated with OS, DSS, and PFI of GC, a risk score models was established: 
Table 1 Major Characteristics of GC patients with multiple clinical prognostic endpoints in TCGA

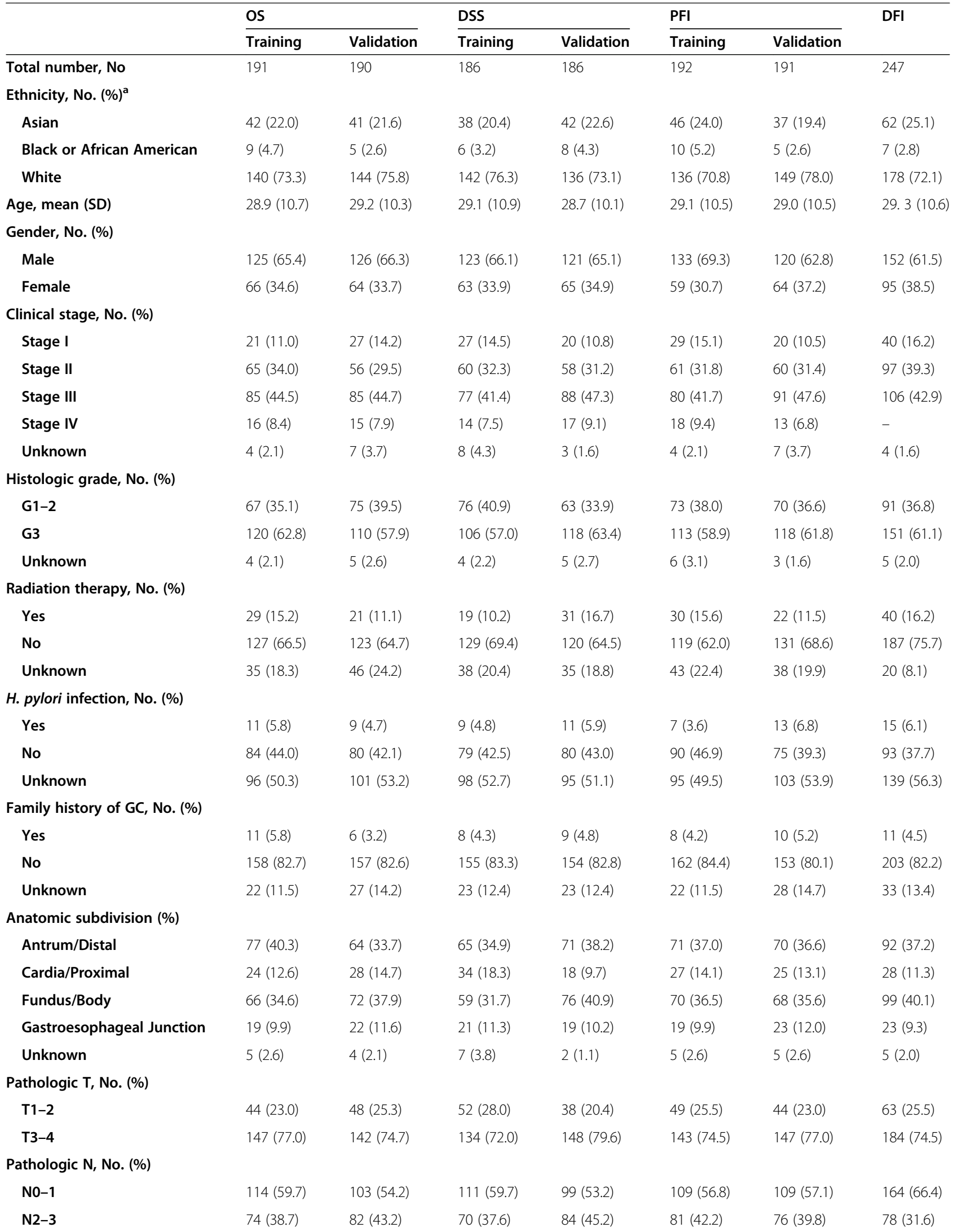


Table 1 Major Characteristics of GC patients with multiple clinical prognostic endpoints in TCGA (Continued)

\begin{tabular}{|c|c|c|c|c|c|c|c|}
\hline & \multicolumn{2}{|l|}{ os } & \multicolumn{2}{|l|}{ DSS } & \multicolumn{2}{|l|}{ PFI } & \multirow[t]{2}{*}{ DFI } \\
\hline & Training & Validation & Training & Validation & Training & Validation & \\
\hline Unknown & $3(1.6)$ & $5(2.6)$ & $5(2.7)$ & $3(1.6)$ & $2(1.0)$ & $6(3.1)$ & $5(2.0)$ \\
\hline \multicolumn{8}{|c|}{ Pathologic M, No. (\%) } \\
\hline Mo & $173(90.6)$ & $169(88.9)$ & $166(89.2)$ & $169(90.9)$ & $171(89.1)$ & $172(90.1)$ & $238(96.4)$ \\
\hline M1 & $9(4.7)$ & $13(6.8)$ & $12(6.5)$ & $10(5.4)$ & $11(5.7)$ & $11(5.8)$ & - \\
\hline Unknown & $9(4.7)$ & $8(4.2)$ & $8(4.3)$ & $7(3.8)$ & $10(5.2)$ & $8(4.2)$ & $9(3.6)$ \\
\hline \multicolumn{8}{|c|}{ Prognostic endpoints, No. (\%) } \\
\hline Event & $77(40.3)$ & $72(37.9)$ & 55 (29.6) & $44(23.7)$ & $77(40.1)$ & $60(31.4)$ & $46(18.6)$ \\
\hline Censored & $114(59.7)$ & $118(62.1)$ & $131(70.4)$ & $142(76.3)$ & $115(59.9)$ & $131(68.6)$ & $201(81.4)$ \\
\hline
\end{tabular}

Abbreviations: GC gastric cancer, OS overall survival, DSS disease-specific survival, DFI disease-free interval, $P F I$ progression-free interval

${ }^{a}$ Missing values of ethnicity were imputed using $R$ package SeSAMe

$$
\begin{aligned}
\text { Model 1 }= & (-0.301) \operatorname{cg} 05275153 \\
& +(-0.436) \operatorname{cg} 10399824 \\
& +(0.375) \operatorname{cg} 11348188 \\
& +(0.348) \operatorname{cg} 11671115 \\
& +(-0.335) \operatorname{cg} 14719951 \\
& +(-0.363) \operatorname{cg} 24406668 \\
& +(0.353) \operatorname{cg} 25117092
\end{aligned}
$$

We also established a risk score model based on the five CpG sites associated with DFI:

$$
\begin{aligned}
\text { Model 2 }= & (-0.301) \operatorname{cg} 05275153 \\
& +(0.375) \operatorname{cg} 11348188 \\
& +(0.348) \operatorname{cg} 11671115 \\
& +(-0.335) \operatorname{cg} 14719951 \\
& +(0.353) \operatorname{cg} 25117092
\end{aligned}
$$

For both models, higher scores indicated worse prognosis potential. After adjusting for all available, potential confounders, both risk scores were independently associated with corresponding clinical endpoints, with a HR $(95 \% \mathrm{CI})$ per one score increase of $1.41(1.24-1.60)$ for OS, $1.70(1.44-1.99)$ for DSS, 1.49 (1.31-1.70) for PFI, and 1.76 (1.30-2.38) for DFI. Kaplan-Meier survival curves showed that two models performed well on predicting OS (log-rank $P=3$ $\left.\times 10^{-7}\right)$, DSS $\left(P=3 \times 10^{-8}\right)$, PFI $\left(P=3 \times 10^{-8}\right)$, and DFI $\left(P=5 \times 10^{-4}\right)$ (Fig. 2). Integrating with all clinicopathologic features, our risk score models achieved the Harrell's concordance index (C-index) of a mean (standard deviation (SD)) of 0.770 (0.019) for OS, $0.794(0.021)$ for DSS, $0.743(0.023)$ for PFI, and 0.784 (0.030) for DFI, which outperformed in prediction compared with models solely utilizing either clinicopathologic features or DNA methylation signatures.

\section{Correlation analysis between DNA methylation and mRNA expression}

For the above highlighted key CpG sites in the gene body, we examined the associations of their DNA methylation level with host gene mRNA expression. Gene body DNA hyper-methylation of cg14719951 (correlation coefficient $=-0.42, P$-value $<2.2 \times 10^{-16}$ ) and cg10399824 (correlation coefficient $=-0.40, P$-value $<$ $2.2 \times 10^{-16}$ ) was inversely associated with mRNA expression of DSC3 and GRK5, respectively. On the contrary, Gene body DNA hyper-methylation of cg25117092 (correlation coefficient $=0.46, P$-value $<2.2 \times 10^{-16}$ ) was significantly associated with increased mRNA expression of $M E D 12 L$. We did not find significant associations between the mRNA expression of these three genes and four clinical prognostic outcomes of GC (Fig. 3).

\section{Discussion}

We comprehensively examined DNA methylation biomarkers associated with multiple prognostic outcomes of GC in an epigenome-wide association analysis based on TCGA. Altered DNA methylation of seven newly reported CpGs was significantly associated with OS, DSS and PFI of GC, including cg10399824 (GRK5), cg05275153 (RGS12), cg24406668 (MMP9), cg14719951(DSC3), and cg25117092 (MED12L), and two in intergenic regions (cg11348188 and cg11671115). Except for cg10399824 and cg24406668, five of them were also significantly associated with DFI of GC. Combining the significant $\mathrm{CpG}$ sites, two risk score models performed well in predicting GC prognosis. Consistent with individual CpG-level associations, DNA methylation of three MCBs involving cg11671115, cg14719951 and cg24406668 was significantly associated with multiple prognostic outcomes of GC.

Genomic location of DNA methylation sites is crucial for determining their potential biological functions [4, 24]. It is well known that aberrant promoter DNA hypermethylation could suppress gene expression [3]. The biological mechanisms of aberrant DNA methylation in intergenic regions or gene body, however, remained overall unclear $[4,25]$. In the current study, cg11348188 and cg11671115 were located in intergenic regions and the other five highlighted $\mathrm{CpG}$ 


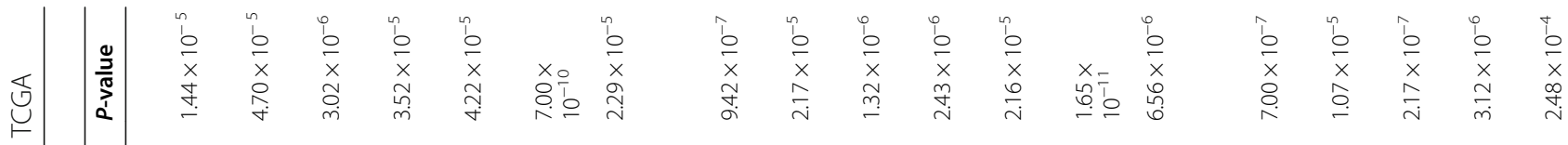

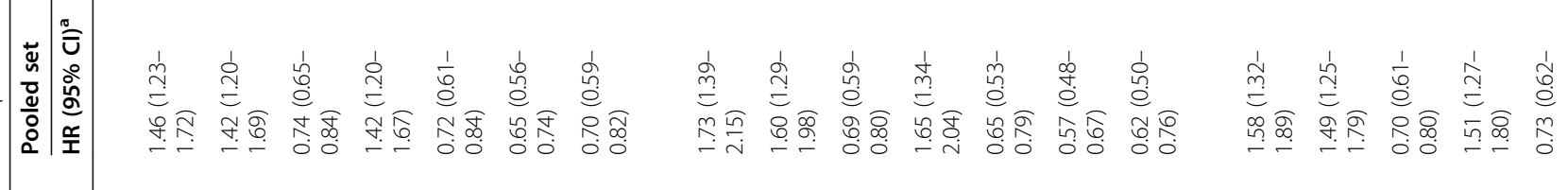

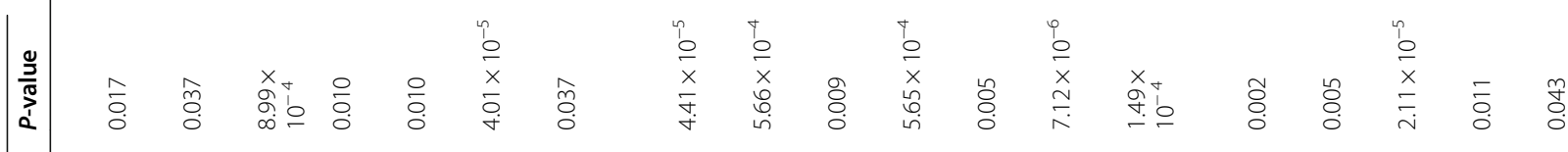

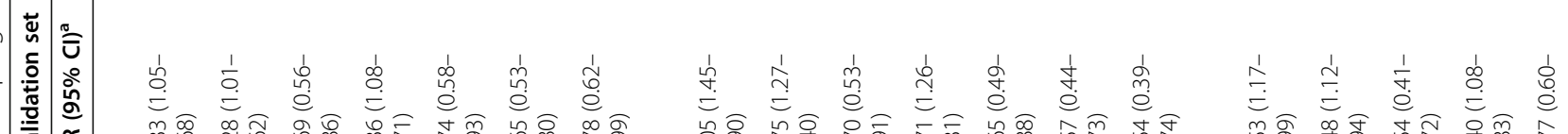

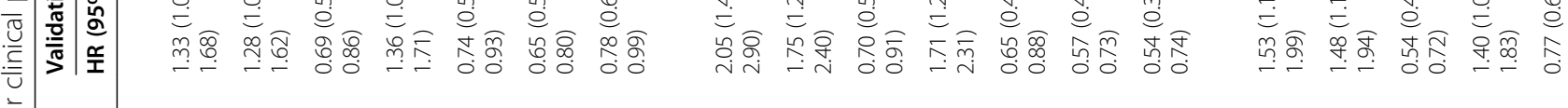

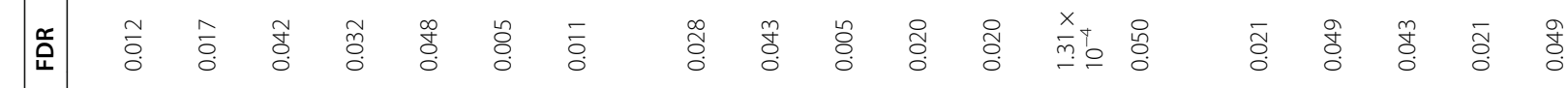

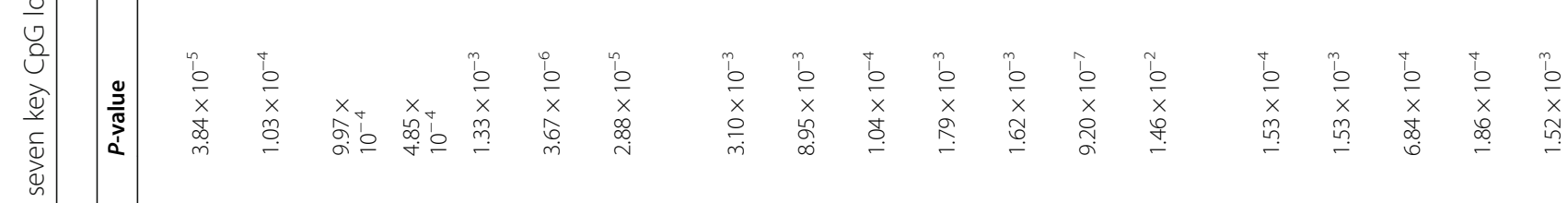

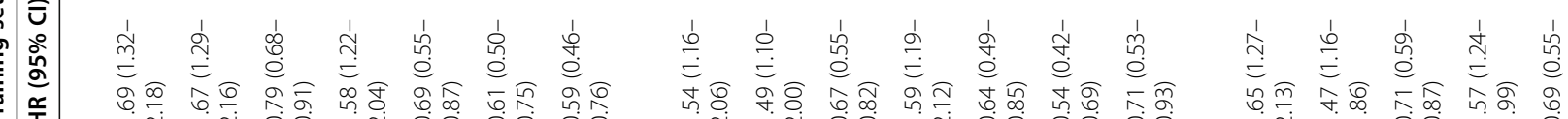

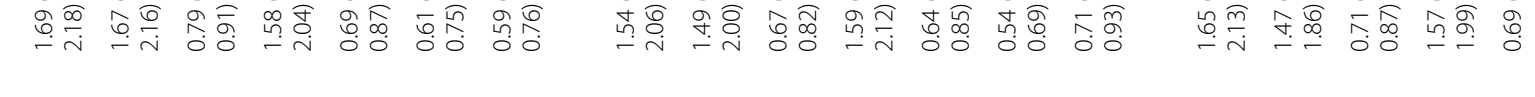

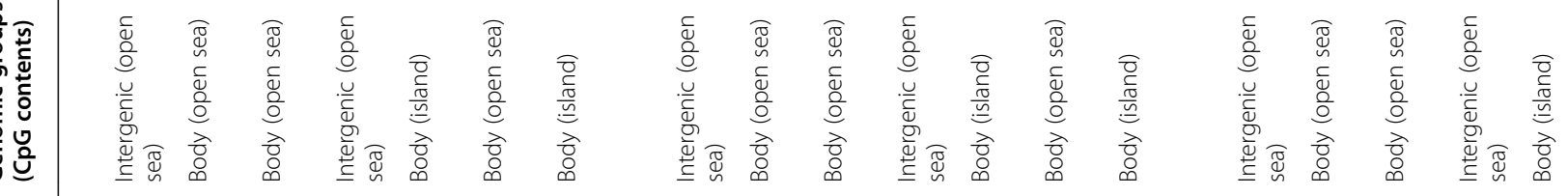

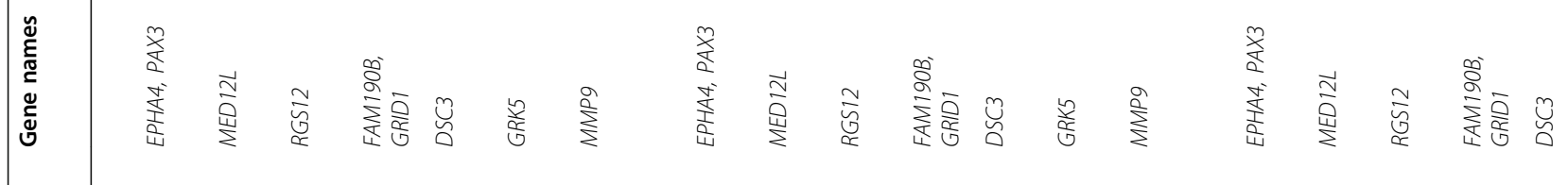




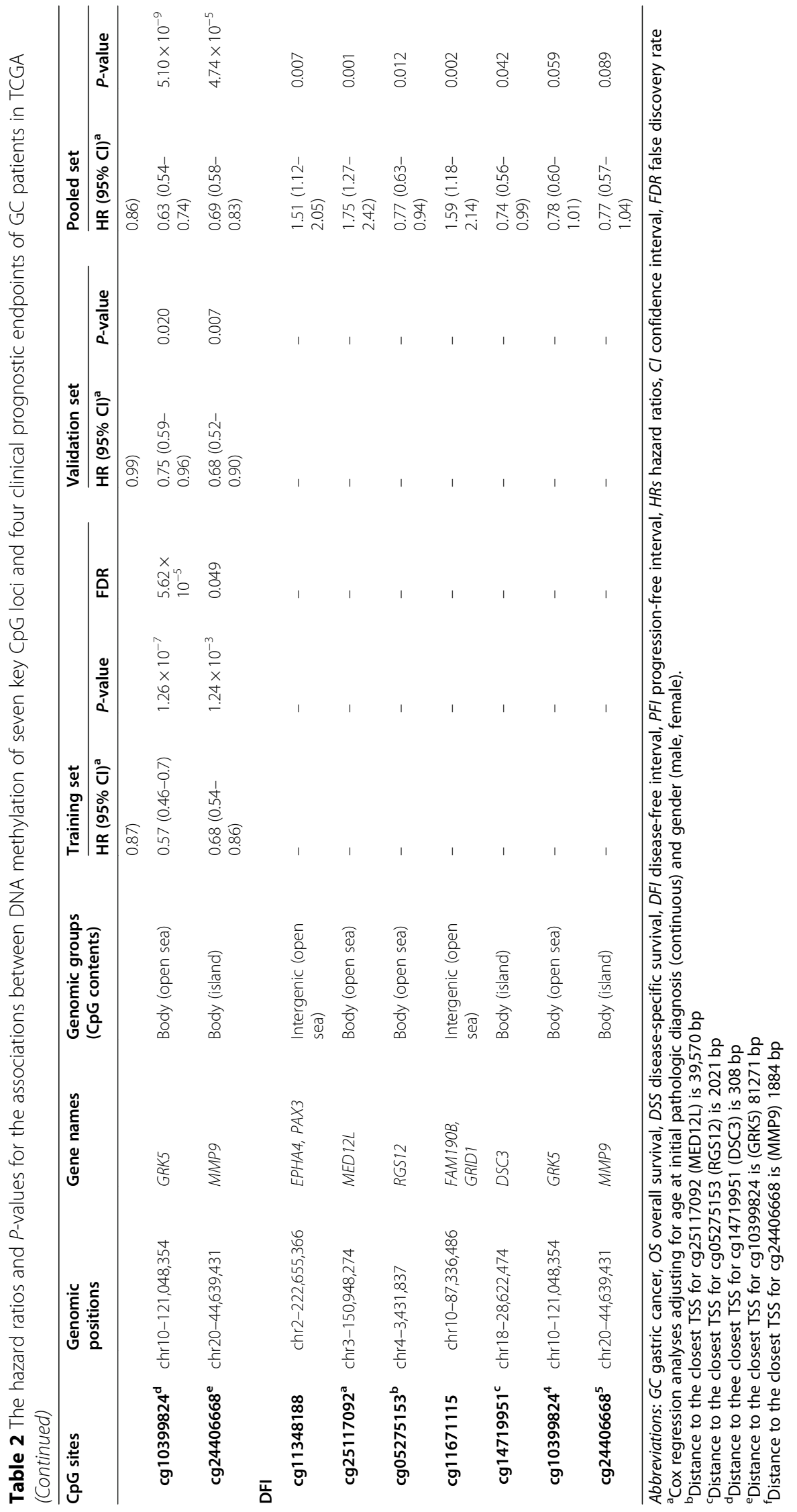


Table 3 Gene set enrichment analysis for gene body DNA methylation in relation to four clinical endpoints of GC patients in TCGA

\begin{tabular}{|c|c|c|c|c|c|c|c|c|c|c|c|}
\hline \multirow[t]{2}{*}{$\overline{\text { ID }}$} & \multirow[t]{2}{*}{ Description } & \multirow[t]{2}{*}{ Number of genes } & \multirow[t]{2}{*}{ Number of unique genes } & \multicolumn{2}{|l|}{ OS } & \multicolumn{2}{|l|}{ DSS } & \multicolumn{2}{|l|}{ PFI } & \multicolumn{2}{|l|}{ DFI } \\
\hline & & & & $\begin{array}{l}P- \\
\text { value }\end{array}$ & FDR & $\begin{array}{l}P \text { - } \\
\text { value }\end{array}$ & FDR & $\begin{array}{l}P- \\
\text { value }\end{array}$ & FDR & $\begin{array}{l}P- \\
\text { value }\end{array}$ & FDR \\
\hline \multicolumn{12}{|l|}{$\overline{\mathrm{GO}^{\mathrm{a}}}$} \\
\hline 35267 & $\begin{array}{l}\text { NuA4 histone } \\
\text { acetyltransferase } \\
\text { complex }\end{array}$ & 31 & 20 & 0.002 & 0.066 & 0.007 & 0.197 & 0.047 & 0.477 & 0.002 & 0.042 \\
\hline 36019 & Endolysosome & 21 & 20 & 0.012 & 0.219 & 0.002 & 0.078 & 0.006 & 0.152 & 0.017 & 0.220 \\
\hline 36020 & $\begin{array}{l}\text { Endolysosome } \\
\text { membrane }\end{array}$ & 14 & 14 & 0.007 & 0.154 & 0.001 & 0.036 & 0.003 & 0.106 & 0.036 & 0.364 \\
\hline 43189 & $\begin{array}{l}\mathrm{H} 4 / \mathrm{H} 2 \mathrm{~A} \text { histone } \\
\text { acetyltransferase } \\
\text { complex }\end{array}$ & 31 & 20 & 0.002 & 0.066 & 0.007 & 0.197 & 0.047 & 0.477 & 0.002 & 0.042 \\
\hline 44346 & $\begin{array}{l}\text { Fibroblast apoptotic } \\
\text { process }\end{array}$ & 24 & 24 & 0.036 & 0.409 & 0.024 & 0.409 & 0.029 & 0.370 & $\begin{array}{l}1.51 \times \\
10^{-4}\end{array}$ & 0.008 \\
\hline \multicolumn{12}{|l|}{ KEGG } \\
\hline 04080 & $\begin{array}{l}\text { Neuroactive ligand- } \\
\text { receptor interaction }\end{array}$ & 272 & 272 & $\begin{array}{l}1.24 \times \\
10^{-16}\end{array}$ & $\begin{array}{l}2.64 \times \\
10^{-14}\end{array}$ & $\begin{array}{l}2.32 \times \\
10^{-16}\end{array}$ & $\begin{array}{l}4.95 \times \\
10^{-14}\end{array}$ & $\begin{array}{l}3.44 \times \\
10^{-10}\end{array}$ & $\begin{array}{l}7.34 \times \\
10^{-8}\end{array}$ & 1.000 & 1.000 \\
\hline 04144 & Endocytosis & 201 & 201 & 0.005 & 0.324 & 0.026 & 0.923 & 0.027 & 0.645 & 0.194 & 0.858 \\
\hline 04950 & $\begin{array}{l}\text { Maturity onset diabetes } \\
\text { of the young }\end{array}$ & 25 & 25 & 0.037 & 0.901 & 0.010 & 0.634 & 0.008 & 0.329 & 1.000 & 1.000 \\
\hline 05217 & Basal cell carcinoma & 55 & 55 & 0.017 & 0.712 & 0.011 & 0.634 & 0.035 & 0.749 & 1.000 & 1.000 \\
\hline
\end{tabular}

sites were located in gene body. Of them, DNA hypermethylation of $M E D 12 L$ was associated with increased mRNA expression, while hypermethylation of DSC3 and GRK5 were associated with decreased mRNA expression. Indeed, previous studies have also reported the complex associations between gene body hypermethylation and increased $[4,26]$ or decreased gene expression [25, 27]. The reason for the opposite directions of findings is unclear. Gene body DNA methylation might increase transcription through blocking the initiation of alternative promoters within gene body or regulating the activities of repetitive DNAs within the transcribed unit [24, 27]. In addition, splicing or elongation of the ordered structure within the transcriptional unit, which was induced by gene body DNA methylation, might also increase transcriptional activity [26]. However, intragenic regions also contain many functional elements, such as enhancers, transcription factor binding cites, and repetitive elements, activation of which induced by aberrant gene body DNA methylation could affect host gene expression. In our study, DNA hypermethylation of RGS12 and MMP9 were not correlated with host gene expression. Interestingly, previous studies on melanoma have reported gene body DNA hypermethylation and increased mRNA expression of $M M P 9$, suggesting possible cell-type specificity of DNA methylation pattern [28, 29].

As aberrant promoter DNA hypermethylation could suppress gene expression, it would be essential to examine the location of five key CpGs in gene body region relative to their closest TSS, so that the effect of altered methylation on gene expression and GC prognosis can be interpreted reasonably. Except for cg1471995, the other four CpGs are located more than 1500 bp to the corresponding closest TSS, indicating that the gene body methylation of these four CpGs may be less likely to affect host gene expression as promoter. For cg1471995, as it was located on the intron 1 of DSC 3 with a distance of $308 \mathrm{bp}$ to the closest TSS, it might act as the promoter regarding its effect on gene expression, which was consistent with our findings that high methylation level of cg14719951 was inversely associated with increased mRNA expression of DSC3. 


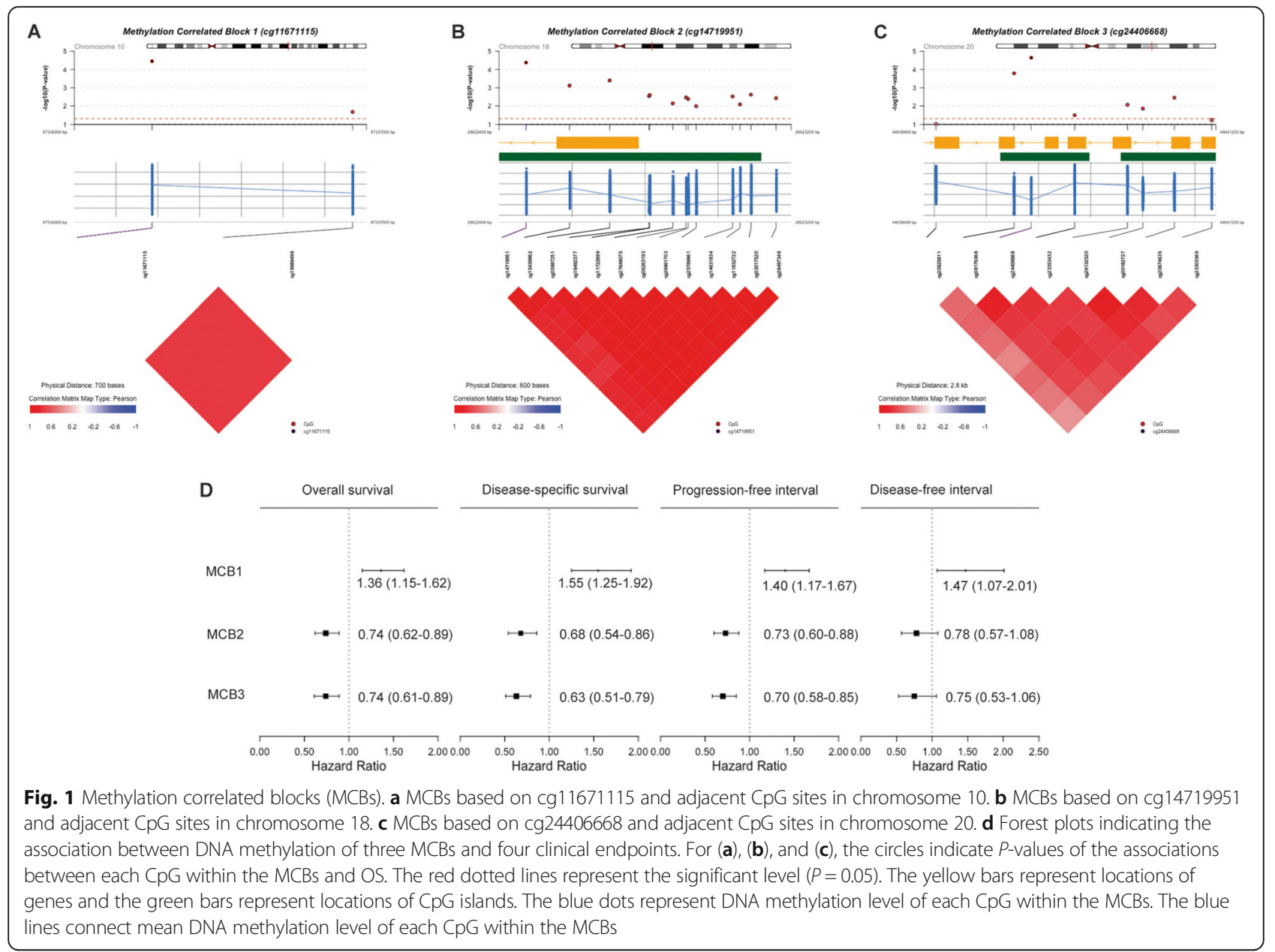

We placed special interests on MED12L, DSC3, and GRK5, the DNA methylation of which was significantly associated with mRNA expression. Somatic mutations and DNA methylation may commonly target the same gene associated with cancer prognosis [30]. MED12L somatic mutations have been identified in both familial and sporadic GC as well as several other cancers [31, 32]. Promoter DNA hypermethylation of DSC3, which suppressed host gene expression, has been associated with poor OS of colorectal cancer and recurrence of prostate cancer [33, 34]. The potential role of GRK5 DNA methylation in the progression/prognosis of cancer has been rarely studied. Interestingly, MED12L, DSC3, and GRK5 mRNA expression was not associated with any GC prognostic outcome in our study. Therefore, gene body DNA methylation might affect GC prognosis not through regulating host gene expression, supporting that the identification of associations between DNA methylation and mRNA expression may not be prerequisites of developing DNA methylation-based biomarkers [4]. In fact, several commercially available DNA methylation-based biomarkers, including DNA methylation of $A P C$ (adenomatosis polyposis coli) and RASSF1 (ras association domain family member 1) as both diagnostic and prognostic biomarkers of prostate cancer and DNA methylation of SEPT9 (septin 9) as predictive biomarker of colorectal cancer, were not correlated with host gene expression [4].

Gene set enrichment analysis revealed that gene body methylation could affect GC prognosis by regulating the neuroactive ligand-receptor interaction pathway [35], which has been also enriched for a number of long noncoding RNAs associated with OS of GC in a prior study [36]. Moreover, this pathway has also been enriched for differentially methylated genes between tumor and normal tissue of GC [37], indicating a significant role in both carcinogenesis and prognosis of GC. Future studies are warranted to clarify how aberrant DNA methylation affect the neuroactive ligand-receptor interaction pathway.

Although DNA methylation of single CpG dinucleotides could effectively regulate host gene expression and are qualified as cancer prognostic biomarkers [38], the pertinent alterations of DNA methylation are 


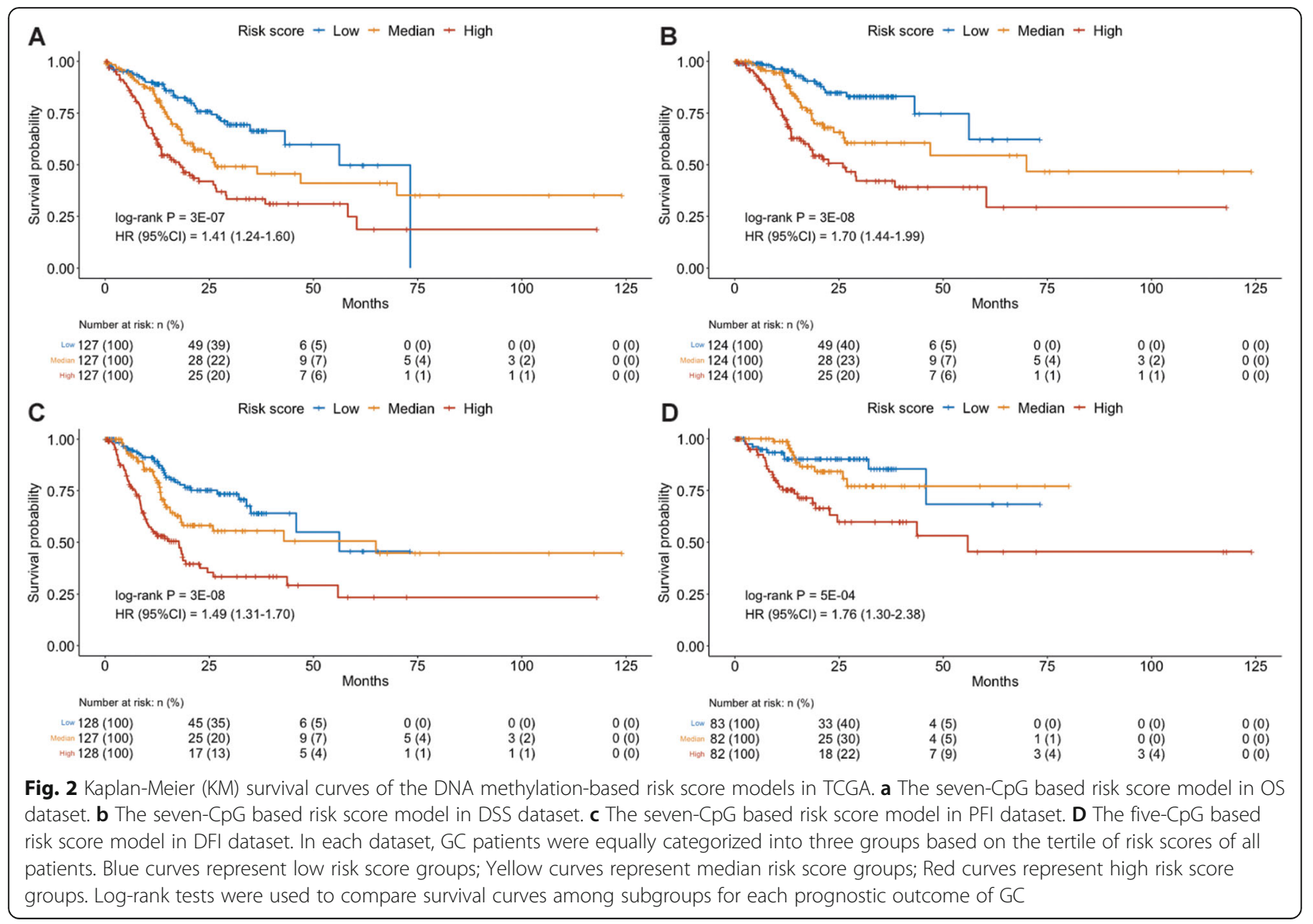

often regional based, with interplays of a number of adjacent CpG sites. Therefore, in addition to identifying the optimal genomic location, defining the optimal number of relevant $\mathrm{CpG}$ sites are of great importance for developing DNA methylation-based biomarker assays [4]. In our study, we also examined DNA methylation patterns accounting for the cluster of adjacent CpG loci. The association of DNA methylation of three identified MCBs with GC prognosis was in line with that based on single $\mathrm{CpG}$ locus, which further strengthened our results.

Based on the publicly available dataset, our study was the first to thoroughly study DNA-methylation based biomarkers associated with multiple prognostic endpoints of GC, including OS, DSS, DFI, and PFI. The association between DNA methylation of seven key CpG loci and GC prognosis was newly reported. The genomic location and the optimal number of adjacent relevant CpG sites were critically evaluated and reported. Two DNA methylation-based risk score models performed well in predicting four clinical outcomes of GC patients. We were able to adjust for the major clinicopathological characteristics in sensitivity analyses, which did not change the results materially.
We acknowledged several limitations. First, DNA methylation profiles in TCGA were measured by Infinium $450 \mathrm{k}$ microarrays, which might restrict us from identifying other uncovered $\mathrm{CpG}$ dinucleotides with biological relevance to GC prognosis. Second, although we tried to adjust for potential confounders, residual confounding might be present. Third, as an exploratory study, both discovery and validation datasets were utilized to identify prognostic $\mathrm{CpG}$ sites and were from the same dataset (TCGA), the performance of our risk score models might be inflated. External datasets with large sample size are warranted to further validate our results in the future. Fourth, our study was an association study based on biostatistical and bioinformatics analyses. Laboratory work is warranted to unravel the biological consequence of these types of DNA methylation.

In conclusion, our study newly identified seven $\mathrm{CpG}$ sites associated with OS, DSS, and PFI of GC, with five of them also associated with DFI of GC. Two DNA methylation-based risk score models were established, which may have implications for clinical practices regarding GC patient stratification of prognosis. Exploration in the experiment setting may contribute to our understanding of the underlying molecular 

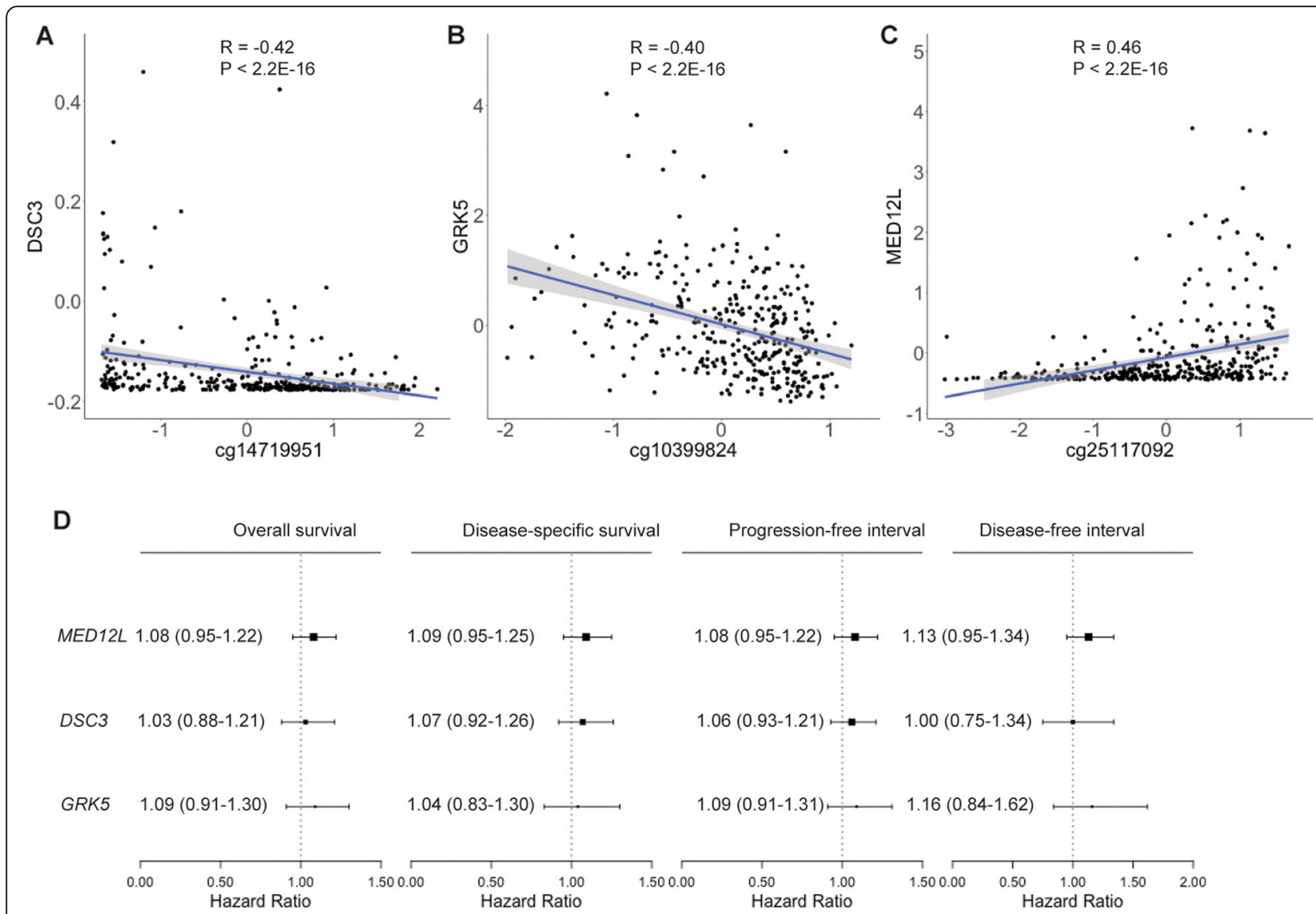

Fig. 3 Spearman correlations between gene body DNA methylation of three CpG loci and host gene expression. a Spearman correlation for cg14719951 (DSC3). b Spearman correlation for cg10399824 (GRK5). c Spearman correlation for cg25117092 (MED12L). d Forest plots indicating the association between mRNA expression of three genes and four clinical endpoints of GC patients

mechanism of gene body DNA methylation on GC prognosis and may inspire the development of novel individualized DNA-methylation based therapeutic strategies.

\section{Supplementary Information}

The online version contains supplementary material available at https://doi. org/10.1186/s12885-021-08389-0.

Additional file 1: Supplementary Table S1. Detailed information on gene set enrichment analyses for gene body DNA methylation in relation to four clinical endpoints of GC patients in TCGA.

\section{Acknowledgements}

We thank all individuals who participated in this study and donated samples.

\section{Authors' contributions}

Jin Dai - study concept and design, acquisition of data, statistical analysis, interpretation of data, drafting of the manuscript, critical revision of the manuscript for important intellectual content; Akihiro Nishi, Zhe-Xuan Li, Yang Zhang, Tong Zhou, Wei-Cheng You - critical revision of the manuscript for important intellectual content; Wen-Qing Li - study concept and design, interpretation of data, critical revision of the manuscript for important intellectual content, funding support, study supervision; Kai-Feng Pan - critical revision of the manuscript for important intellectual content, funding support, study supervision. The author(s) read and approved the final manuscript.

\section{Funding}

This work was supported by Beijing Talents Foundation (2018000021223ZK01), Michigan Medicine-PKUHSC Joint Institute for Translational and Clinical Research (BMU2020J|004), the Fundamental Research Funds for the Central Universities, Capital's Funds for Health Improvement and Research (CFH 2020-2-1026), Beijing Municipal Administration of Hospitals' Ascent Plan (DFL20181102).

\section{Availability of data and materials}

The datasets analyzed during the current study are available in the Genomic Data Commons Data Portal of National Cancer Institute (https://portal.gdc. cancer.gov/).

\section{Declarations}

\section{Ethics approval and consent to participate}

Inform consent was waived by the Institutional Review Board of Peking University Cancer Hospital as this study was based on a publicly accessible dataset.

Consent for publication

Not applicable.

\section{Competing interests}

The authors declare no potential conflicts of interest.

\section{Author details}

${ }^{1}$ Key Laboratory of Carcinogenesis and Translational Research (Ministry of Education/Beijing), Department of Cancer Epidemiology, Peking University 
Cancer Hospital \& Institute, 52 Fucheng Rd, Haidian District, Beijing 100142, People's Republic of China. ${ }^{2}$ Department of Epidemiology, UCLA Fielding School of Public Health, Los Angeles, CA 90095, USA. ${ }^{3}$ Joint International Research Center of Translational and Clinical Research, Beijing 100142, China.

\section{Received: 3 February 2021 Accepted: 19 May 2021}

Published online: 25 May 2021

\section{References}

1. Bray F, Ferlay J, Soerjomataram I, Siegel RL, Torre LA, Jemal A. Global cancer statistics 2018: GLOBOCAN estimates of incidence and mortality worldwide for 36 cancers in 185 countries. CA Cancer J Clin. 2018;68(6):394-424. https://doi.org/10.3322/caac.21492.

2. The global, regional, and national burden of stomach cancer in 195 countries, 1990-2017: a systematic analysis for the Global Burden of Disease study 2017. Lancet Gastroenterol Hepatol. 2020;5:42-54. https://doi.org/10.1 016/s2468-1253(19)30328-0.

3. Das PM, Singal R. DNA methylation and cancer. J Clin Oncol. 2004;22(22): 4632-42. https://doi.org/10.1200/jco.2004.07.151.

4. Koch A, Joosten SC, Feng Z, de Ruijter TC, Draht MX, Melotte V, et al. Analysis of DNA methylation in cancer: location revisited. Nat Rev Clin Oncol. 2018;15(7):459-66. https://doi.org/10.1038/s41571-018-0004-4.

5. Peng $Y$, Wu Q, Wang L, Wang $H$, Yin F. A DNA methylation signature to improve survival prediction of gastric cancer. Clin Epigenetics. 2020;12(1):15. https://doi.org/10.1186/s13148-020-0807-x.

6. Bai Y, Wei C, Zhong Y, Zhang Y, Long J, Huang S, et al. Development and validation of a prognostic nomogram for gastric Cancer based on DNA methylation-driven differentially expressed genes. Int J Biol Sci. 2020;16(7): 1153-65. https://doi.org/10.7150/ijbs.41587.

7. Liu D, Ma X, Yang F, Xiao D, Jia Y, Wang Y. Discovery and validation of methylated-differentially expressed genes in Helicobacter pylori-induced gastric cancer. Cancer Gene Ther. 2019;27(6):473-85. https://doi.org/10.1038/ s41417-019-0125-7.

8. Li C, Zheng Y, Pu K, Zhao D, Wang Y, Guan Q, et al. A four-DNA methylation signature as a novel prognostic biomarker for survival of patients with gastric cancer. Cancer Cell Int. 2020;20(1):88. https://doi.org/10.1186/s12935020-1156-8.

9. Hudis CA, Barlow WE, Costantino JP, Gray RJ, Pritchard KI, Chapman JAW, et al. Proposal for standardized definitions for efficacy end points in adjuvant breast cancer trials: the STEEP system. J Clin Oncol. 2007;25(15): 2127-32. https://doi.org/10.1200/jco.2006.10.3523.

10. Chibaudel B, Bonnetain F, Shi Q, Buyse M, Tournigand C, Sargent DJ, et al. Alternative end points to evaluate a therapeutic strategy in advanced colorectal cancer: evaluation of progression-free survival, duration of disease control, and time to failure of strategy--an aide et Recherche en Cancerologie digestive group study. J Clin Oncol. 2011;29(31):4199-204. https://doi.org/10.1200/jco.2011.35.5867.

11. Liu J, et al. An Integrated TCGA Pan-Cancer Clinical Data Resource to Drive High-Quality Survival Outcome Analytics. Cell. 2018;173:400-416.e411. https://doi.org/10.1016/j.cell.2018.02.052

12. Colaprico A, Silva TC, Olsen C, Garofano L, Cava C, Garolini D, et al. TCGAbiolinks: an R/Bioconductor package for integrative analysis of TCGA data. Nucleic Acids Res. 2016;44(8):e71. https://doi.org/10.1093/nar/gkv1507.

13. Aryee MJ, Jaffe AE, Corrada-Bravo H, Ladd-Acosta C, Feinberg AP, Hansen $K D$, et al. Minfi: a flexible and comprehensive Bioconductor package for the analysis of Infinium DNA methylation microarrays. Bioinformatics. 2014; 30(10):1363-9. https://doi.org/10.1093/bioinformatics/btu049.

14. Triche TJ Jr, Weisenberger DJ, Van Den Berg D, Laird PW, Siegmund KD. Low-level processing of Illumina Infinium DNA methylation BeadArrays. Nucleic Acids Res. 2013;41(7):e90. https://doi.org/10.1093/nar/gkt090.

15. Zhou W, Triche TJ Jr, Laird PW, Shen H. SeSAMe: reducing artifactual detection of DNA methylation by Infinium BeadChips in genomic deletions. Nucleic Acids Res. 2018;46:e123. https://doi.org/10.1093/nar/gky691.

16. Dai J, et al. Whole Genome Messenger RNA Profiling Identifies a Novel Signature to Predict Gastric Cancer Survival. Clin Transl Gastroenterol. 2019; 10:e00004. https://doi.org/10.14309/ctg.0000000000000004.

17. Glickman ME, Rao SR, Schultz MR. False discovery rate control is a recommended alternative to Bonferroni-type adjustments in health studies. J Clin Epidemiol. 2014;67(8):850-7. https://doi.org/10.1016/j.jclinepi.2014.03. 012.
18. Kanehisa M, Araki M, Goto S, Hattori M, Hirakawa M, Itoh M, et al. KEGG for linking genomes to life and the environment. Nucleic Acids Res. 2008; 36(Database):D480-4. https://doi.org/10.1093/nar/gkm882.

19. Gene Ontology Consortium: going forward. Nucleic Acids Res. 2015;43: D1049-56. https://doi.org/10.1093/nar/gku1179.

20. Khatri P, Sirota M, Butte AJ. Ten years of pathway analysis: current approaches and outstanding challenges. PLoS Comput Biol. 2012;8(2): e1002375. https://doi.org/10.1371/journal.pcbi.1002375.

21. Ren X, Kuan P. F. methylGSA: a Bioconductor package and shiny app for DNA methylation data length bias adjustment in gene set testing. Bioinformatics. 2019;35(11):1958-9. https://doi.org/10.1093/bioinformatics/ bty892.

22. Hao X, Luo H, Krawczyk M, Wei W, Wang W, Wang J, et al. DNA methylation markers for diagnosis and prognosis of common cancers. Proc Natl Acad Sci U S A. 2017;114(28):7414-9. https://doi.org/10.1073/pnas.1703577114.

23. Martin TC, Yet I, Tsai PC, Bell JT. coMET: visualisation of regional epigenomewide association scan results and DNA co-methylation patterns. BMC Bioinformatics. 2015;16:131. https://doi.org/10.1186/s12859-015-0568-2.

24. Jones PA. Functions of DNA methylation: islands, start sites, gene bodies and beyond. Nat Rev Genet. 2012;13(7):484-92. https://doi.org/10.1038/ nrg3230.

25. Kulis M, Heath S, Bibikova M, Queirós AC, Navarro A, Clot G, et al. Epigenomic analysis detects widespread gene-body DNA hypomethylation in chronic lymphocytic leukemia. Nat Genet. 2012;44(11):1236-42. https:// doi.org/10.1038/ng.2443.

26. Yang X, Han H, de Carvalho DD, Lay FD, Jones PA, Liang G. Gene body methylation can alter gene expression and is a therapeutic target in cancer. Cancer Cell. 2014;26(4):577-90. https://doi.org/10.1016/j.ccr.2014. 07.028 .

27. Maunakea AK, Nagarajan RP, Bilenky M, Ballinger TJ, D'Souza C, Fouse SD, et al. Conserved role of intragenic DNA methylation in regulating alternative promoters. Nature. 2010;466(7303):253-7. https://doi.org/10.1038/ nature09165.

28. Falzone $L$, et al. MMP-9 overexpression is associated with intragenic hypermethylation of MMP9 gene in melanoma. Aging (Albany NY). 2016;8: 933-44. https://doi.org/10.18632/aging.100951.

29. Kristensen LS, Raynor MP, Candiloro I, Dobrovic A. Methylation profiling of normal individuals reveals mosaic promoter methylation of cancerassociated genes. Oncotarget. 2012;3:450-61. https://doi.org/10.18632/ oncotarget.480.

30. Chan TA, Glockner S, Yi JM, Chen W, van Neste L, Cope L, et al. Convergence of mutation and epigenetic alterations identifies common genes in cancer that predict for poor prognosis. PLoS Med. 2008;5(5):e114 https://doi.org/10.1371/journal.pmed.0050114.

31. Majewski IJ, Kluijt I, Cats A, Scerri TS, de Jong D, Kluin RJC, et al. An a-Ecatenin (CTNNA1) mutation in hereditary diffuse gastric cancer. J Pathol. 2013;229(4):621-9. https://doi.org/10.1002/path.4152.

32. Barbieri CE, Baca SC, Lawrence MS, Demichelis F, Blattner M, Theurillat JP, et al. Exome sequencing identifies recurrent SPOP, FOXA1 and MED12 mutations in prostate cancer. Nat Genet. 2012;44(6):685-9. https://doi.org/1 $0.1038 /$ ng.2279

33. Cui T, Chen Y, Yang L, Knösel T, Zöller K, Huber O, et al. DSC3 expression is regulated by p53, and methylation of DSC3 DNA is a prognostic marker in human colorectal cancer. Br J Cancer. 2011;104(6):1013-9. https://doi.org/1 0.1038/bjc.2011.28.

34. Pan J, Chen Y, Mo C, Wang D, Chen J, Mao X, et al. Association of DSC3 mRNA down-regulation in prostate cancer with promoter hypermethylation and poor prognosis. PLoS One. 2014;9(3):e92815. https://doi.org/10.1371/ journal.pone.0092815.

35. Kim HJ, Kang TW, Haam K, Kim M, Kim SK, Kim SY, et al. Whole genome MBD-seq and RRBS analyses reveal that hypermethylation of gastrointestinal hormone receptors is associated with gastric carcinogenesis. Exp Mol Med. 2018;50(12):1-14. https://doi.org/10.1038/ s12276-018-0179-X.

36. Su X, Zhang J, Yang W, Liu Y, Liu Y, Shan Z, et al. Identification of the prognosis-related IncRNAs and genes in gastric Cancer. Front Genet. 2020; 11:27. https://doi.org/10.3389/fgene.2020.00027.

37. Li H, Liu JW, Liu S, Yuan Y, Sun LP. Bioinformatics-based identification of methylated-differentially expressed genes and related pathways in gastric Cancer. Dig Dis Sci. 2017;62(11):3029-39. https://doi.org/10.1007/s10620-01 7-4740-6. 
38. Claus R, Lucas DM, Stilgenbauer S, Ruppert AS, Yu L, Zucknick M, et al. Quantitative DNA methylation analysis identifies a single CpG dinucleotide important for ZAP-70 expression and predictive of prognosis in chronic lymphocytic leukemia. J Clin Oncol. 2012;30(20):2483-91. https://doi.org/1 0.1200/jco.2011.39.3090.

\section{Publisher's Note}

Springer Nature remains neutral with regard to jurisdictional claims in published maps and institutional affiliations.

Ready to submit your research? Choose BMC and benefit from:

- fast, convenient online submission

- thorough peer review by experienced researchers in your field

- rapid publication on acceptance

- support for research data, including large and complex data types

- gold Open Access which fosters wider collaboration and increased citations

- maximum visibility for your research: over $100 \mathrm{M}$ website views per year

At BMC, research is always in progress.

Learn more biomedcentral.com/submissions 